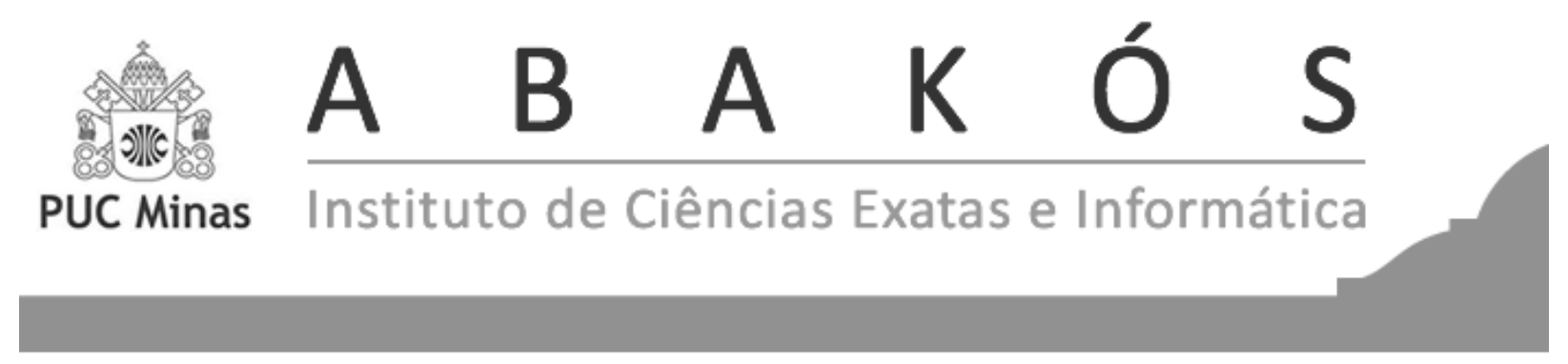

\title{
Ensino de Números Complexos no Ensino Médio, Técnico e Superior: um Mapeamento de Produções Brasileiras*
}

Teaching Complex Numbers in High School, Technical and Higher Education: a Mapping of Brazilian Productions

\author{
Cassiano Scott Puhl ${ }^{1}$ \\ Isolda Gianni de Lima ${ }^{2}$ \\ Thaísa Jacintho Müller ${ }^{3}$
}

\begin{abstract}
Resumo
Este artigo apresenta um mapeamento teórico de artigos que abordam o ensino de números complexos no Ensino Médio, Técnico e Superior, publicados em periódicos brasileiros. Este estudo teve como objetivo identificar como os números complexos são abordados no Ensino Superior, por meio de recursos digitais, buscando reconhecer recursos disponíveis, verificar diferentes concepções, metodologias de pesquisas e abordagens didáticas planejadas ou aplicadas. Os artigos foram selecionados de periódicos de qualis A1, A2 e B1, da área de ensino do quadriênio 2013-2016. A análise realizada revela que os números complexos são considerados um conhecimento prévio pelos professores de Engenharia e que os acadêmicos, no entanto, não apresentam esse conhecimento. Nas pesquisas consideradas no mapeamento predominam ou a problemática de números complexos ou de circuitos elétricos. Portanto, o mapeamento apresentado neste trabalho evidencia a necessidade de investigar essa temática e a pertinência de propor uma alternativa pedagógica para auxiliar professores e acadêmicos.
\end{abstract}

Palavras-chave: Números complexos. Mapeamento teórico. Ensino. Aprendizagem.

\footnotetext{
*submetido em: 22/01/2020 - aceito em: 09/11/2020.

${ }^{1}$ Doutorando do Programa de Pós-Graduação em Educação em Ciências e Matemática da Pontifícia Universidade Católica do Rio Grande do Sul - c.s.puhl@hotmail.com

${ }^{2}$ Doutora em Informática na Educação pela Universidade Federal do Rio Grande do Sul. Professora da Universidade de Caxias do Sul nos cursos de Engenharia e Licenciatura em Matemática - thaisamuller@gmail.com

${ }^{3}$ Doutora em Informática na Educação pela Universidade Federal do Rio Grande do Sul. Professora da Pontifícia Universidade Católica do Rio Grande do Sul (PUCRS), atuando na Escola Politécnica e como professora permanente do programa de Pós Graduação em Educação em Ciências e Matemática - iglima@ucs.br
} 


\begin{abstract}
This work presents a theoretical mapping of articles that address the teaching of complex numbers in High School, Technical, and Higher Education, published in Brazilian journals. This study aimed to identify how complex numbers are approached in higher education, seeking to recognize the available resources, verify different conceptions, research methodologies, and planned or applied approaches. The articles were selected from journals of categories A1, A2, and B1, in the teaching area of the 2013-2016 quadrennium. The analysis carried out reveals that complex numbers are considered prior knowledge by engineering professors and that academics, however, do not have this knowledge. In research considered in the mapping, the problem of complex numbers or electrical circuits predominates. Therefore, the mapping presented in this work shows the need to investigate this topic and the relevance of proposing a pedagogical alternative to assist teachers and academics.
\end{abstract}

Keywords: Complex numbers. Theoretical mapping. Teaching. Learning. 


\section{INTRODUÇÃO}

As reformas educacionais que vem sendo implantadas no Brasil - Pacto Nacional pelo Fortalecimento do Ensino Médio (BRASIL, 2013), Ensino Médio Inovador (BRASIL, 2014) e, recentemente, a criação da Base Nacional Comum Curricular (BRASIL, 2018) - foram propostas com o propósito de melhorar a educação. No entanto, essa expectativa não tem sido alcançada. Os índices de diferentes avaliações externas comprovam que houveram poucos avanços e nisso está a ruptura da cultura educacional - de um ensino meramente expositivo e uma aprendizagem mecânica - que já deveria ter acontecido.

A Base Nacional Comum Curricular (BNCC) é a proposta de mudança mais recente e visa efetivar e promover essa transformação. A BNCC foi planejada em consonância com os princípios das Diretrizes Curriculares Nacionais da Educação Básica, indicando competências e habilidades consideradas fundamentais para a formação dos estudantes da Educação Básica (BRASIL, 2018). Dos conhecimentos matemáticos, nessa nova demarcação de conteúdos escolares, alguns deixam de ser indicados como fundamentais do Ensino Médio como, por exemplo, os números complexos (BRASIL, 2018).

Os números complexos já costumavam ser abdicados em muitas escolas, principalmente do ensino público (BATISTA, 2004; MELLO; SANTOS, 2005; ELI, 2014; PEREIRA, 2016; PUHL, 2016; COSTA, 2016), o que pode ter colaborado para que não constassem nos conteúdos propostos pela BNCC. Porém, a falta desse conhecimento tem impactado a Educação Superior (COELHO, 2013), causando dificuldades, principalmente, para os acadêmicos de Engenharia Elétrica, que necessitam destas noções, por exemplo, para simplificar o processo da análise dos componentes de circuitos elétricos em corrente alternada (REIS, 2009; STUMP; ABAR, 2013; LIRA, 2014; JESUS; TESTANI, 2016; GERMANO, 2016; AGRICCO JUNIOR, 2017; LINHARES, 2017; LIMA, 2017; PORTOLAN, 2017; OLIVEIRA, 2018).

Diante disso, buscou-se informações em pesquisas e em produtos educacionais para compreender as dificuldades, nos processos de ensino e de aprendizagem de números complexos no Ensino Superior, e estratégias didáticas utilizadas para superá-las, de modo a auxiliar professores e acadêmicos de Engenharia. Assim, este estudo caracteriza-se como um mapeamento teórico cujo objetivo é identificar como os números complexos são abordados no Ensino Superior, por meio de recursos digitais, buscando reconhecer recursos disponíveis, verificar diferentes concepções, metodologias de pesquisas e abordagens didáticas planejadas ou aplicadas.

Segundo Biembengut (2008), as pesquisas de mapeamento teórico justificam-se por proporcionar aos leitores informações para compreender um contexto a ser investigado, ou ainda, de reconhecer e mapear os conhecimentos produzidos em lugares e tempos distintos por outros pesquisadores, permitindo estabelecer relações ou identificar questões ainda não reconhecidas ou investigadas. Seguindo as orientações propostas por Biembengut (2008) para se realizar esse mapeamento, o presente artigo foi estruturado em quatro etapas: conceitos e definições; identificação; classificação e organização; e reconhecimento e/ou análise. 


\section{CONCEITOS E DEFINIÇÕES}

Na primeira etapa, conceitos e definições, apresentam-se os termos envolvidos na presente investigação. O mapeamento teórico pode ser utilizado como princípio metodológico na pesquisa educacional, tendo várias funções: “[...] reconhecer os mais diversos fatores que se manifestam sobre os entes pesquisados; entender um fato, uma questão dentro de um cenário; servir-se do conhecimento produzido e reordenar alguns setores deste conhecimento" (BIEMBENGUT, 2008, p. 135).

No presente estudo o mapeamento tem o objetivo de revelar conhecimentos, ou seja, trazer informações para auxiliar os professores e acadêmicos de Engenharia a qualificarem os processos de ensino e de aprendizagem no Ensino Superior. Contudo, se o mapeamento não atingir seu objetivo, devido à insuficiência de informações e de pesquisas com essa temática, justifica-se a realização de uma investigação, pois uma pesquisa "[...] é requerida quando não se dispõe de informação suficiente para responder ao problema, ou então quando a informação disponível se encontra em tal estado de desordem que não possa ser adequadamente relacionada ao problema" (GIL, 2017, p. 1).

No caso de o mapeamento não revelar conhecimentos com potencial para auxiliar os processos de ensino e de aprendizagem, contemplará outros objetivos, como

[...] entender um fato, uma questão dentro de um cenário, servir do conhecimento produzido e reordenar alguns setores deste conhecimento. Quanto mais nos inteiramos dos entes e dos diversos fatores que levam à resultante, mais nos habilitamos em aplicar conhecimentos e, por recorrência, mais conhecimentos dispomos para construir um mapa que ainda não existe, para situar, contextualizar a pesquisa de forma a mostrar, descrever, narrar, circunscrever o problema, explicando e justificando sua legitimidade (BIEMBENGUT, 2008, p. 136).

Sendo assim, o mapeamento teórico possibilita que a comunidade acadêmica dê continuidade à pesquisa ou aprimore um já existente (BIEMBENGUT, 2008). Em relação à delimitação do mapeamento das pesquisas acadêmicas, Biembengut (2008) e Creswell (2010) destacam a preferência por uma literatura recente, pois, teoricamente, nessas pesquisas já teriam considerado conhecimentos de outras mais antigas. Biembengut (2008) sugere considerar pesquisas dos últimos cinco anos e Creswell (2010) dos últimos 10 anos. No entanto, considerando-se a perspectiva de Alves-Mazzotti e Gewandsznajder (1999) - da pouca realização da revisão da literatura por pesquisadores brasileiros -, optou-se por selecionar produções de periódicos com qualis é A1, A2 e B1, da área de ensino do quadriênio 2013-2016. Nesse processo, não se considerou o ano de publicação do artigo, selecionando todas disponíveis virtualmente, que contemplassem os critérios definidos no campo de busca dos sites dos periódicos. 


\section{IDENTIFICAÇÃO}

Nessa etapa realiza-se o levantamento de pesquisas que podem contribuir na atual investigação, optando-se por selecionar artigos de periódicos com qualis é A1, A2 e B1, da área de ensino do quadriênio 2013-2016. Como há diferentes periódicos, de diferentes áreas de conhecimento, inicialmente considerou-se as áreas de Matemática, Ciência e Informática na Educação, com buscas na Plataforma Sucupira ${ }^{4}$ das seguintes palavras/expressões: "Matemática", "Ciência" e "Tecnologia ou informática", totalizando 57 o número de periódicos encontrados. Após essa busca, acessou-se o site de cada periódico com a expressão "números complexos", que resultou em 62 artigos, sendo 48 de periódicos da área de Matemática (MAT), 12 de Ciências (CIE) e um de Informática na Educação (INF_ED). O Quadro 1 apresenta os periódicos encontrados com o respectivo qualis e a quantidade de artigos sobre números complexos.

Quadro 1 - Artigos sobre números complexos em periódicos qualis A1, A2 e B1

\begin{tabular}{|c|c|c|c|}
\hline Área & Periódico & Qualis & Quantidade \\
\hline MAT & Bolema & A1 & 23 \\
\hline MAT & Revista Brasileira de História da Matemática & B1 & 12 \\
\hline CIE & Revista Brasileira de Ensino de Ciência e Tecnologia & $\mathrm{A} 2$ & 9 \\
\hline MAT & Educação Matemática em Revista & A2 & 3 \\
\hline MAT & $\begin{array}{l}\text { Educação e Matemática: Revista da Associação de Pro- } \\
\text { fessores }\end{array}$ & B1 & 2 \\
\hline CIE & $\begin{array}{l}\text { Revista Electrónica de Investigación en Educación en Ci- } \\
\text { encias }\end{array}$ & A2 & 1 \\
\hline MAT & Acta Scientiae & A2 & 1 \\
\hline MAT & $\begin{array}{l}\text { Amazônia: Revista de Educação em Ciências e Matemá- } \\
\text { ticas }\end{array}$ & $\mathrm{A} 2$ & 1 \\
\hline MAT & Educação Matemática em Revista-RS & A2 & 1 \\
\hline MAT & $\begin{array}{l}\text { PNA: Revista de Investigación en Didáctica de la Mate- } \\
\text { mática }\end{array}$ & A2 & 1 \\
\hline MAT & Revista Dynamis & A2 & 1 \\
\hline MAT & $\begin{array}{l}\text { RELIME: Revista Latinoamericana de Investigacion en } \\
\text { Matematica Educativa }\end{array}$ & A2 & 1 \\
\hline MAT & Vidya & A2 & 1 \\
\hline CIE & Experiências em Ensino de Ciências & B1 & 1 \\
\hline CIE & $\begin{array}{l}\text { Tecné, Episteme y Didaxis: Revista de la Facultad De Ci- } \\
\text { encia y Tecnologia }\end{array}$ & B1 & 1 \\
\hline INF_ED & Renote: Revista Novas Tecnologias na Educação & B1 & 1 \\
\hline MAT & Perspectivas da Educação Matemática & B1 & 1 \\
\hline MAT & Tendências em Matemática Aplicada e Computacional & B1 & 1 \\
\hline CIE & Ensaio. Pesquisa em Educação em Ciências & A1 & 0 \\
\hline CIE & Enseñanza de las Ciencias & A1 & 0 \\
\hline CIE & Revista de Educacion de las Ciencias & A1 & 0 \\
\hline CIE & $\begin{array}{l}\text { Revista Eureka sobre Enseñanza y Divulgación de Las Ci- } \\
\text { encias }\end{array}$ & A1 & 0 \\
\hline
\end{tabular}

${ }^{4}$ Disponível em: https://sucupira.capes.gov.br/sucupira/. Acesso em: 03 nov. 2019. 


\begin{tabular}{|c|c|c|c|}
\hline Área & Periódico & Qualis & Quantidade \\
\hline CIE & Revista Areté: Revista Amazônica de Ensino de Ciências & A2 & 0 \\
\hline CIE & Anais da Academia Brasileira de Ciências & A2 & 0 \\
\hline CIE & Enseñanza de las Ciencias de la Tierra & A2 & 0 \\
\hline CIE & Investigações em Ensino de Ciências & A2 & 0 \\
\hline CIE & Revista Electrónica de Enseñanza de las Ciencias & A2 & 0 \\
\hline CIE & Revista Brasileira de Pesquisa em Educação em Ciências & A2 & 0 \\
\hline INF_ED & Revista Latinoamericana de Tecnología Educativa & $\mathrm{A} 2$ & 0 \\
\hline MAT & $\begin{array}{l}\text { Alexandria: Revista de Educação em Ciência e Tecnolo- } \\
\text { gia }\end{array}$ & A2 & 0 \\
\hline MAT & Educação Matemática Pesquisa & $\mathrm{A} 2$ & 0 \\
\hline MAT & Jornal Internacional de Estudos em Educação Matemática & A2 & 0 \\
\hline MAT & $\begin{array}{l}\text { REDIMAT: Revista de Investigación en Didáctica de las } \\
\text { Matemáticas }\end{array}$ & A2 & 0 \\
\hline MAT & Revista de Ensino de Ciências e Matemática & A2 & 0 \\
\hline MAT & REVEMAT: Revista Eletrônica de Educação Matemática & A2 & 0 \\
\hline MAT & Revista de Educação, Ciências e Matemática & $\mathrm{A} 2$ & 0 \\
\hline MAT & Zetetike & A2 & 0 \\
\hline MAT & REVEDUC & A2 & 0 \\
\hline $\mathrm{CIE}$ & Archivos de Ciencias de la Educación & B1 & 0 \\
\hline CIE & Gondola: Ensenanza y Aprendizaje de las Ciencias & B1 & 0 \\
\hline CIE & Ensino de Ciências e Tecnologias em Revista & B1 & 0 \\
\hline CIE & Revista Ciências \& Ideias & B1 & 0 \\
\hline CIE & Revista de Ciências da Educação & B1 & 0 \\
\hline CIE & Unopar Científica. Ciências Humanas e Educação & B1 & 0 \\
\hline CIE & Ciência \& Ensino & B1 & 0 \\
\hline CIE & Revista Brasileira de Educação em Ciência da Informação & B1 & 0 \\
\hline CIE & Revista Ciência e Tecnologia & B1 & 0 \\
\hline $\mathrm{CIE}$ & Tear: Revista de Educação, Ciência e Tecnologia & B1 & 0 \\
\hline INF_ED & Revista Educação \& Tecnologia & B1 & 0 \\
\hline INF_ED & Revista Iberoamericana de Ciencia Tecnología y Sociedad & B1 & 0 \\
\hline INF_ED & Revista Tecnologia e Sociedade & B1 & 0 \\
\hline INF_ED & Tecnologia Educacional & B1 & 0 \\
\hline INF_ED & Revista Tecnologias na Educação & B1 & 0 \\
\hline INF_ED & Informática na Educação & B1 & 0 \\
\hline MAT & Boletim Online de Educação Matemática & B1 & 0 \\
\hline MAT & $\begin{array}{l}\text { Em Teia: Revista de Educação Matemática e Tecnológica } \\
\text { Iberoamericana }\end{array}$ & B1 & 0 \\
\hline MAT & Revista Paranaense de Educação Matemática & B1 & 0 \\
\hline
\end{tabular}

Fonte: Elaborado pelos autores com dados da busca.

Em virtude do número elevado de artigos, realizou-se a leitura dos resumos e das introduções, verificando quais trabalhos poderiam colaborar para qualificar os processos de ensino e de aprendizagem de números complexos, categorizando-se os artigos conforme o tema abordado e iniciando-se, assim, a etapa da classificação e organização. 


\section{CLASSIFICAÇÃO E ORGANIZAÇÃO}

Segundo Biembengut (2008, p. 93), na classificação e organização busca-se "[...] identificar os pontos relevantes ou significativos que nos valham como guia para compreender os segmentos já pesquisados e expressos de forma a nos permitir elaborar um sistema de explicação ou de interpretação". Mediante a leitura dos resumos, conforme consta na Tabela 1, foram selecionados os trabalhos para o estudo atual, pois no título da maioria dos artigos não consta a expressão números complexos, sendo mais comum encontrá-la descrita no resumo ou no decorrer do texto.

Tabela 1 - Categorias de temas de pesquisas que abordam números complexos

\begin{tabular}{l|c}
\hline \multicolumn{1}{c|}{ Tema } & Quantidade \\
\hline A - Estudos cuja problemática não são números complexos & 45 \\
\hline $\begin{array}{l}\text { B - Construção de um objeto de aprendizagem para o ensino de números com- } \\
\text { plexos }\end{array}$ & 4 \\
\hline C - Estratégias didáticas com geometria dinâmica ou outros recursos digitais & 3 \\
\hline D - Estratégias didáticas sem a utilização de recursos digitais & 3 \\
\hline $\begin{array}{l}\text { E - Estudos de revisão de literatura sobre as contribuições de matemáticos ou } \\
\text { evolução histórica de números complexos }\end{array}$ & 2 \\
\hline $\begin{array}{l}\text { F - Estudo de revisão de literatura sobre aplicações de números complexos em } \\
\text { disciplinas de Matemática }\end{array}$ & 2 \\
\hline $\begin{array}{l}\text { G - Estratégias didáticas de aplicação de números complexos em circuitos elé- } \\
\text { tricos em corrente alternada }\end{array}$ & 1 \\
\hline H - Conceitos incompreendidos sobre números complexos & 1 \\
\hline $\begin{array}{l}\text { I - Estudo de revisão de literatura de números complexos em disciplinas de } \\
\text { Sistema de Sinais }\end{array}$ & 1 \\
\hline
\end{tabular}

Fonte: Elaborada pelos autores.

A categoria A refere-se a pesquisas cujo objeto de investigação não contempla tais números, mas esses são citados ou apresentados como exemplo em estudos sobre outro tema. Esse mapeamento produziu ainda informações sobre a evolução histórica dos números complexos, encontrada como objeto de pesquisa ou como fundamentação teórica, que compuseram a categoria E.

Contemplando o ensino de números complexos com tecnologias tem-se a categoria $\mathrm{B}$, com trabalhos em que os autores relatam a construção de objeto de aprendizagem (OA) para promover aprendizagens de estudantes do Ensino Médio ou Ensino Médio Técnico. Já na categoria $\mathrm{C}$ constam as produções com estratégias didáticas apoiadas por recursos digitais, para estudantes de Ensino Médio.

Em ensino, ainda se tem as categorias D e G com trabalhos que, respectivamente, abordam estratégias didáticas sem a utilização de recursos digitais e com atividades em que os números complexos são utilizados para analisar circuitos elétricos em corrente alternada, especialmente no Ensino Médio Técnico em Eletrotécnica e Eletrônica. Outra categoria com reflexos no ensino de números complexos é a dos "Conceitos incompreendidos sobre números 
complexos", em que o pesquisador verifica quais conceitos e operações que os licenciados de Matemática não compreendem sobre o tema.

Além de produções voltadas ao ensino, há outras inspiradas em revisão de literatura sobre a aplicação de números complexos no estudo de variáveis complexas na disciplina de Cálculo ou em Sistema de Sinais para acadêmicos de Engenharia estabelecendo respectivamente as categorias F e I.

Estabelecidas as categorias, leram-se as produções acadêmicas referentes aos temas: "Estratégias didáticas com geometria dinâmica ou outros recursos digitais", "Estratégias didáticas de aplicação de números complexos em circuitos elétricos em corrente alternada", "Construção de um objeto de aprendizagem para o ensino de números complexos", "Conceitos incompreendidos sobre números complexos" e "Estudo de revisão de literatura sobre aplicações de números complexos em Matemática". A escolha desses temas justificou-se pela possibilidade de as pesquisas fornecerem informações e conhecimentos consonantes com a problemática investigada. Desse modo, o mapeamento tem potencial para atingir seus objetivos: “[...] compreender os fatos, ponderá-los, compará-los, rejeitar alguns, conservar outros, reunir elementos que possam vir a se constituir em excepcional embasamento ao pesquisador." (BIEMBENGUT, 2008, p. 95).

Por meio do mapeamento delimitaram-se as produções acadêmicas de interesse, totalizando 10 artigos. O Quadro 2 foi elaborado para apresentar, em ordem cronológica, detalhes sobre as referências bibliográficas dos textos selecionados, sendo possível identificar o autor, o título da produção, o periódico, o ano de publicação e em que categoria a pesquisa se enquadrou (EA: Estudo de revisão de literatura sobre aplicações de números complexos em Matemática; SD: Estratégias didáticas com geometria dinâmica ou outros recursos digitais; COA: Construção de um objeto de aprendizagem para o ensino de números complexos; SDA: Estratégias didáticas de aplicação de números complexos em circuitos elétricos em corrente alternada; e D: Conceitos incompreendidos sobre números complexos). 


\section{Quadro 2 - Produções acadêmicas selecionadas para o mapeamento}

\begin{tabular}{|c|c|c|}
\hline $\mathrm{N}^{\mathrm{o}}$ & Referência & Categoria \\
\hline PE1 & $\begin{array}{l}\text { MELLO, Sílvio Quintino de; SANTOS, Renato Pires dos. O ensino } \\
\text { de Matemática e a educação profissional: a aplicabilidade dos números } \\
\text { complexos na análise de circuitos elétricos. Acta Scientiae, Canoas/RS, } \\
\text { v. } 7 \text {, n. } 2 \text {, jul./dez. } 2005 \text {. }\end{array}$ & SDA \\
\hline PE2 & $\begin{array}{l}\text { PARDO, Tomás; GÓMEZ, Bernardo. La enseñanza y el aprendizaje } \\
\text { de los números complejos: un estudio en el nivel universitário. PNA } \\
\text { - Revista de Investigación en Didáctica de la Matemática, Granada - } \\
\text { Espanha, v. } 2 \text {, n. } 1 \text {, jan. } 2007 \text {. }\end{array}$ & $\mathrm{D}$ \\
\hline PE3 & $\begin{array}{l}\text { BATISTA, Silvia Cristina Freitas; BARCELOS, Gilmara Teixeira; } \\
\text { COSTA Débora Maciel da; BEHAR, Patricia Alejandra. Investigando } \\
\text { em C: uma unidade de aprendizagem online para estudo de números } \\
\text { complexos. RENOTE - Revista Novas Tecnologias na Educação, Porto } \\
\text { Alegre/RS, v. 7, n. 1, jul. } 2009 \text {. }\end{array}$ & $\mathrm{COA}$ \\
\hline PE4 & $\begin{array}{l}\text { MONZON, Larissa Weyh; GRAVINA, Maria Alice. Uma Introdução } \\
\text { às Funções de Variável Complexa no Ensino Médio: uma possibilidade } \\
\text { através do uso de animações interativas. Bolema, Rio Claro/SP, v. } 27 \text {, } \\
\text { n. } 46 \text {, ago. } 2013 \text {. }\end{array}$ & $\mathrm{COA}$ \\
\hline PE5 & $\begin{array}{l}\text { ABEGG, Ilse; RAMOS, Diego Berlezi. Investigação de ferramentas e } \\
\text { métodos de ensino de circuitos de corrente alternada para curso intro- } \\
\text { dutório de eletrotécnica. Revista Dynamis, Blumenau/SC, v. 19, n. 1, } \\
2013 \text {. }\end{array}$ & SD \\
\hline PE6 & $\begin{array}{l}\text { BITENCOURT, Agner Lopes; VARGAS, Paulo Roberto; FELICETTI, } \\
\text { Vera Lucia. Una propuesta pedagógica: utilizando el software Geoge- } \\
\text { bra en la rotación de vectores complejos. Amazônia - Revista de Edu- } \\
\text { cação em Ciências e Matemáticas, Belém/PA, v. 11, n. 21, jul./dez. } \\
2014 \text {. }\end{array}$ & SD \\
\hline PE7 & $\begin{array}{l}\text { PINTO, José Eustáquio; LAUDARES, João Bosco. Objeto de Apren- } \\
\text { dizagem de Números Complexos com aplicações na área técnica em } \\
\text { eletroeletrônica. Revista Brasileira de Ensino de Ciência e Tecnologia, } \\
\text { Ponta Grossa/PR, v. 9, n. 3, maio/ago. } 2016 \text {. }\end{array}$ & $\mathrm{COA}$ \\
\hline PE8 & $\begin{array}{l}\text { ALVES, Francisco Regis Vieira. Transição complexa do cálculo - TCC: } \\
\text { engenharia didática para as noções de sequências, séries e série de po- } \\
\text { tências. Educação Matemática em Revista-RS, Canos/RS, v. 1, n. 17, } \\
2016 \text {. }\end{array}$ & EA \\
\hline PE9 & $\begin{array}{l}\text { PUHL, Cassiano Scott; LIMA, Isolda Gianni de. Números Complexos: } \\
\text { interação e aprendizagem. Educação Matemática em Revista, Brasíli- } \\
\text { a/DF, v. 22, n. 55, jul./set. } 2017 \text {. }\end{array}$ & $\mathrm{COA}$ \\
\hline PE10 & $\begin{array}{l}\text { PUHL, Cassiano Scott; LIMA, Isolda Gianni de. Interagindo com os } \\
\text { números complexos: revivendo o problema das raízes sofistas. Pers- } \\
\text { pectivas da Educação Matemática, Campo Grande/MS, v. 10, n. 24, } \\
\text { set./dez. } 2017 \text {. }\end{array}$ & SD \\
\hline
\end{tabular}

Fonte: Elaborado pelos autores com dados da busca.

Na sequência, concomitante à leitura dos artigos, produziu-se uma síntese ${ }^{5}$, com aspec-

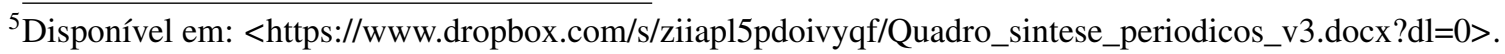
Acesso em 16 nov. 2018. 
tos convergentes e divergentes aos propósitos deste estudo, destacando-se contribuições para compreender a problemática investigada.

\section{RECONHECIMENTO E ANÁLISE}

Em relação aos tipos de produções acadêmicas, constataram-se seis pesquisas aplicadas, sendo três para estudantes de nível médio técnico (PE1, PE5 e PE7), duas para o nível superior (PE3 e PE6) e duas para nível médio (PE4 e PE9). Nas produções PE1, PE3, PE4, PE7 e P9, os autores construíram objetos de aprendizagem para ensinar os números complexos, sendo que PE3, PE4 e PE9 deram ênfase para as propriedades matemáticas e PE1 e PE7 para a aplicação de números complexos na análise de circuitos elétricos em corrente alterada, sendo assim, para conhecimento da área da Elétrica.

Os trabalhos relatados nos artigos PE3, PE4, PE9 e P10 desenvolveram os OA denominados, respectivamente, Investigando em C, Números Complexos ${ }^{6}$ e Número Complexos: interação e aprendizagem ${ }^{7}$, sendo que somente o último ainda se encontra disponível na web. Os desenvolvedores de ambos os OA criaram aplicativos no GeoGebra que permitem relacionar os conhecimentos algébricos com os geométricos, ampliando as possibilidades de qualificar os processos de ensino e de aprendizagem de números complexos para professores e licenciandos de Matemática, no caso da PE3 e para estudantes de Ensino Médio nas PE4 e PE9.

$\mathrm{Na} \mathrm{PE} 3$, os professores avaliaram o nível de dificuldade das atividades sugeridas, sendo que pouco mais da metade dos participantes consideraram "moderado" e os demais "fácil". Para os autores, a justificativa para considerarem o nível de dificuldade "moderado" consiste na pouca utilização de atividades investigativas em sala de aula e à predominância do modelo considerado tradicional: de abordagem teórica, baseada no livro didático, explicação de exemplos e proposição de exercícios para serem resolvidos, na maioria das vezes similares aos exemplos e exercícios demonstrativos apresentados pelo professor. Além disso, um aspecto relevante desta pesquisa é o fato de que os participantes avaliaram o $\mathrm{OA}$ em relação à usabilidade, conforme pode ser observado na Figura 1, e destaca-se que dos artigos analisados esse é o único que apresenta alguma avaliação pelos usuários do OA proposto.

Diferente de PE3, o estudo PE4 apresenta um OA para o ensino de funções de variáveis complexas, visando introduzir esse conteúdo no Ensino Médio. Além de aplicativos criados no GeoGebra, os autores disponibilizaram recortes da coletânea de vídeos "Dimensions: une promenade mathematique”, com explicações sobre números complexos e funções de variáveis complexas, explorando seus significados em processos de criação de fractais. Como ênfase da pesquisa, foi destacada a interação com o GeoGebra, pois os estudantes manipulavam e exploraram a animação de acordo com seu interesse, curiosidade ou dificuldade, transitando entre registros algébricos e geométricos, o que conduziu à aprendizagem de situações simples a mais elaboradas. Em busca de evidenciar a aprendizagem, foi criado um ambiente denominado "Para

${ }^{6}$ Disponível em: <http://www.ufrgs.br/espmat/disciplinas/numeros_complexos/>. Acesso em: 10 out. 2020.

${ }^{7}$ Disponível em: <http://numeroscomplexos.online/>. Acesso em: 10 out. 2020. 


\section{Figura 1 - Avaliação do OA pelos participantes da PE3}

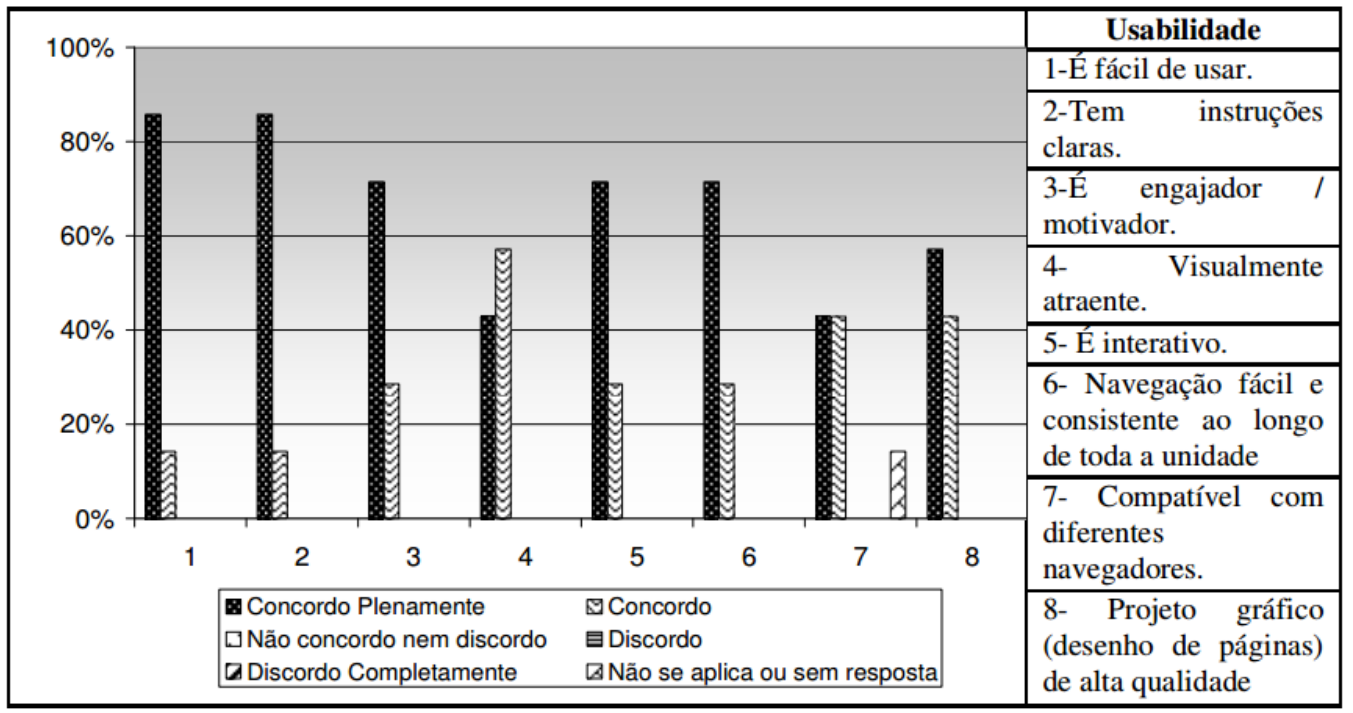

Fonte: (BATISTA et al., 2009, p. 9).

Pensar", que apresenta ao estudante um conjunto de questões a serem exploradas e resolvidas. Após o experimento com a utilização do OA, os autores concluíram que o ambiente "Para Pensar" mostrou-se propício para o desenvolvimento de uma aprendizagem com autonomia. Além disso, citam que com o OA é possível ampliar o universo das funções que podem ser estudadas na escola, podendo-se abordar na Educação Básica os processos interativos com funções de variável complexa, como um contexto natural de aplicação dos números complexos, dando origem às apreciadas figuras dos fractais.

No estudo PE10 apresenta-se o OA Números Complexos: interação e aprendizagem, construído com base em pesquisas realizadas com professores do Ensino Básico e do Ensino Superior, por meio da leitura de trabalhos científicos, da participação em eventos de Educação Matemática e uma perspectiva própria de estratégias para o ensino e a aprendizagem de números complexos. O OA Números Complexos: interação e aprendizagem é composto de dez espaços de aprendizagem: Caminhada histórica, Espaço do vestibulando, Fazer e compreender, Apoio tecnológico, Rotas de Aprendizagem, Quem quer dinheiro? Show do Milhão, Foco na teoria, Calculadora, Aplicações e Fórum de discussões. Esses espaços são compostos de textos didáticos, vídeos informativos, questões de diversos vestibulares, sequências de atividades e aplicativos de GeoGebra que propiciam aos estudantes, por manipulação e interação, a construção de conhecimentos de forma ativa e significativa.

Apesar do PE10 não apresentar resultados empíricos, os autores, no trabalho PE9, propõem a utilização de um espaço de aprendizagem do Números Complexos: interação e aprendizagem, para introduzir conceitos de números complexos, por meio de uma abordagem história. Nessa abordagem, os estudantes são desafiadores a resolverem seguinte o problema: "Diga dois números cuja soma é 6 , e a multiplicação é 13". Os estudantes são guiados e orientados por um personagem chamado Radice, o qual faz o papel de professor, envolvendo-os no processo de resolução e na aprendizagem de conhecimentos novos que o desafio c ontempla. Além de 
resolver o desafio, os estudantes comprovaram que os resultados, número complexos, satisfazem as condições do desafio. Por fim, os autores concluem que essa abordagem não se limitou a apresentar informações históricas, mas propiciou a "vivência" de dificuldades similares as encontradas pelos matemáticos do século XVI, permitindo com que os estudantes construíssem conceitos matemáticos.

De outro modo, a problemática da PE7 dá ênfase à análise de circuitos elétricos em corrente alternada, em um experimento aplicado a 20 estudantes do segundo ano do Curso Técnico em Equipamentos Biomédicos, da área de Eletroeletrônica, do Centro Federal de Educação Tecnológica de Minas Gerais, que interagiram com o OA Descomplicando os complexos. O OA é proposto para desenvolver uma aprendizagem autônoma dos números complexos com aplicações na área técnica em eletroeletrônica, mediada por um conjunto de seis atividades: as duas primeiras destacam a relação algébrica e geométrica dos números complexos, associando-os com vetores, tendo como objetivo a aprendizagem das propriedades matemáticas; e as quatro seguintes envolvem a aplicação dos conceitos e das operações com números complexos na análise de circuitos elétricos.

Nesse estudo, PE7, os dados foram analisados com método de Análise de Erro, sendo definidas quatro categorias em relação aos erros identificados nas resoluções dos estudantes:

- Erro na compreensão do enunciado: erros relacionados a uma má interpretação do enunciado do item e dos dados presentes no enunciado da atividade disponibilizada na tela do Objeto de Aprendizagem.

- Incompreensão do ambiente informatizado: erros relacionados à dificuldade de manusear ou entender as ferramentas que dinamizam o enunciado dos itens da atividade.

- Erros operacionais: são erros relacionados à defasagem de conteúdo, relacionados à manipulação algébrica ou desatenção nos passos de resolução.

- Erros de compatibilidade: erros relacionados à falta de coerência da resposta com os dados dos itens da atividade.

Por fim, os autores do PE7 relatam que o OA Descomplicando os Complexos propiciou interação entre o professor, os estudantes e as atividades propostas, além de estimular a realização de experimentações e simulações. Como resultado, os estudantes exploraram e formalizaram as propriedades relacionadas às operações, a partir da movimentação de pontos e vetores, e compreenderam a representação geométrica das operações com números complexos.

Com mais ênfase no ensino, PE1, PE5, PE6 apresentam estratégias didáticas, sendo que PE1 e PE5 para o Ensino Médio Técnico em Eletromecânica e PE6 para o Ensino Médio. Os participantes da aplicação de PE1 foram professores do Ensino Médio Técnico do Rio Grande do Sul, e o objetivo foi investigar a aplicação dos números complexos como estratégia de ensino na análise de circuitos elétricos em corrente alternada. Nesse estudo, houve duas turmas experimentais que utilizaram a análise complexa - com números complexos -, enquanto uma turma de controle utilizou a análise fasorial - sem os números complexos. Após a aplicação 
da estratégia didática, os autores entrevistaram os professores participantes, verificando que a análise complexa é mais usada pelos professores que possuem maior titulação, ao contrário da análise fasorial que é adotada por professores que pouco avançaram no caminho acadêmico.

Em relação ao ensino da análise de circuitos elétricos em corrente alternada, os pesquisadores do PE1 concluíram que 51,4\% dos professores entrevistados utilizam a análise fasorial; $32,4 \%$ utilizam diferentes métodos; $13,5 \%$ fazem uso da análise complexa; e uma minoria, $2,7 \%$ utiliza softwares de simulações. Os resultados mostraram que as tecnologias ainda são pouco exploradas e utilizadas pelos professores. Uma explicação para o baixo percentual da utilização da análise complexa, segundo, praticamente, metade os professores, é o fato de que no ensino de números complexos não são apresentadas aplicações. Em outras justificativas, aparece com alguma expressão, que os estudantes não estudaram ou não compreenderam os números complexos.

Contudo, os autores destacam que a análise fasorial apresenta limitações, sendo impraticável, principalmente, na análise de circuitos mistos. O conhecimento de números complexos facilita e permite que sejam explorados circuitos com um número maior de componentes, sendo assim um pré-requisito aos estudantes da disciplina de Eletricidade.

Em uma perspectiva similar, o trabalho PE5 consiste em uma estratégia didática para os estudantes da disciplina de Eletrotécnica-II do curso Técnico em Eletromecânica do Colégio Técnico Industrial de Santa Maria, que utiliza recursos digitais para auxiliar na compreensão dos conceitos relacionados aos circuitos elétricos em corrente alternada. Segundo os autores, desenvolver tais conhecimentos, na maioria das vezes, implica em dificuldades, pois o estudante precisa relacionar elementos matemáticos, como números complexos, conceitos de geometria e noções de grandezas fatoriais com conhecimentos da Física mais avançada, tais como leis do eletromagnetismo ou produção de energia elétrica. Para auxiliar nos processos de ensino e de aprendizagem, os autores utilizam três recursos digitais: um vídeo disponível no YouTube; um aplicativo, em $J A V A$, que permite ao estudante manipular elementos sobre as características de um gerador elementar de corrente alternada; e um software de simulação, chamado PSIM, que consiste em uma ferramenta computacional mais ampla, não gratuita, pode ser utilizada em disciplinas que abordam eletrônica, máquinas elétricas, fontes alternativas, dentre outras.

Em outro enfoque, os autores da PE6 destacam a importância de abordar os números complexos por meio de diferentes representações, conforme é proposto pela Teoria de Representação Semiótica ${ }^{8}$ de Raymond Duval, integrando álgebra e geometria. Nesse estudo foram propostos quatro exercícios de rotação no plano Argand-Gauss, resolvidos com o auxílio do GeoGebra, para 12 licenciandos de Matemática do Centro Universitário La Salle na disciplina de Variáveis Complexas. Além das resoluções dos exercícios, os autores analisaram dois livros didáticos do Ensino Médio: Matemática de Manoel Paiva, publicado em 1999, e Matemática, Contexto e Aplicações de Luiz Roberto Dante, publicado em 2012, e concluíram que sua organi-

\footnotetext{
${ }^{8}$ A semiótica é definida como a "ciência dos signos", tratando assim do estudo das linguagens, sejam verbais ou não verbais (CALDEIRA, 2013). Duval (2003) afirma que o aprendizado matemático ocorre nas transformações de representações, existindo quatro tipos de registros de representação: língua natural, sistemas de escritas, figurais e gráficos.
} 
zação considera um modelo de aula dita tradicional: apresenta-se a teoria, seguida de exemplos resolvidos e por fim há uma lista de exercícios propostos, sem destacar as diferentes representações dos números complexos e sem proporcionar atividades interativas ou investigativas.

Como resultado desse estudo, os autores do PE6 concluíram que a utilização do GeoGebra permitiu estudar e visualizar os efeitos de cada operação, considerando a representação dos números complexos como vetores, que contribuiu principalmente para o entendimento da multiplicação, operação que é responsável pela rotação, somando o valor dos argumentos para o vetor resultante, e aumentando ou diminuindo o módulo desse vetor. Da análise, concluíram ser pertinente estudar os números complexos relacionando propriedades algébricas com geométricas, conforme também foi relatado nos artigos PE3, PE4 e PE7.

Dessas pesquisas aplicadas, somente a estratégia didática da PE6 foi proposta a estudantes que nunca haviam estudado os números complexos. Os autores descrevem a estratégia, mas não apresentam detalhes da sua aplicação em sala de aula. Nas demais pesquisas, os participantes já tinham algum conhecimento e os objetivos incluíam ampliar ou qualificar o aprendizado sobre os números complexos, em termos matemáticos ou físicos, ou verificar o nível de conhecimento ou de dificuldade dos participantes.

As pesquisas, PE2 e PE8, selecionadas a priori, não têm implicação direta no ensino de conceitos introdutórios de números complexos. Os autores da PE8 discutem o ensino do conteúdo de sequências, séries e séries de potências de números complexos para os acadêmicos da disciplina de Cálculo, utilizando o GeoGebra e fundamentando-a na Teoria das Situações Didáticas e ressaltando a importância das variáveis complexas na Engenharia. Nessa pesquisa, o GeoGebra foi utilizado para ilustrar a representação geométrica de séries e sequências. Contudo, os autores apresentam a resolução de alguns exemplos no GeoGebra, mas não mostram e nem sugerem como tais representações poderiam ser aplicadas aos acadêmicos.

Já no artigo PE2 é verificado o nível de conhecimento sobre números complexos de 19 licenciandos de Matemática da Universidade de Valencia. Para isso, foi aplicado um questionário composto de cinco questões, das quais somente quatro foram apresentadas e discutidas. Os dados da pesquisa PE2 evidenciam que os licenciandos pouco sabem de números complexos. Somente cinco resolveram corretamente uma equação de $2^{\circ}$ grau com discriminante negativo, sendo que a maioria afirmou que não era possível resolver, pois não existe raiz quadrada de número negativo. Complementando esse cenário, ninguém reconheceu a existência de um logaritmo de número negativo, pois para a maioria dos licenciandos só existe logaritmo de números positivos. Na multiplicação, menos da metade dos licenciandos resolveu corretamente a operação com números complexos e ninguém relatou a impossibilidade de ordenar os números complexos. Por fim, os autores concluíram que o ensino de números complexos não considera as dificuldades e inconsistências que estiveram presentes ao longo da evolução e da história da Matemática, com isso os estudantes reproduzem esse cenário, não compreendendo os conceitos e as operações com os números complexos. 


\section{CONSIDERAÇÕES FINAIS}

ste trabalho apresentou um recorte sobre o tema de números complexos, em que se analisaram artigos publicados nos 58 periódicos mapeados, de qualis A1, A2 e B1 na área de ensino do quadriênio 2013-2016. Os resultados do mapeando poderiam ser outros se tivessem sido considerados outros critérios ou mais periódicos.

Nesse estudo destacou-se a existência de publicações sobre o ensino de números complexos aplicados na análise de circuitos elétricos em corrente alternada, sendo considerado um conhecimento prévio na perspectiva dos professores que participaram da pesquisa do artigo PE1, pois assim podem-se estudar circuitos elétricos mistos, com um número maior de componentes. Além disso, confirmou-se a importância de saber números complexos para aprender circuitos elétricos em corrente alternada, principalmente por proporcionar a compreensão de conceitos mais inclusivos, o que seria impraticável ao utilizar a análise fasorial.

Em uma perspectiva similar, em PE5, ficou evidenciado que os estudantes apresentam dificuldade na aprendizagem de circuitos elétricos em corrente alternada, principalmente de relacionar elementos matemáticos, dentre esses os números complexos, conceitos de geometria e noções de grandezas fatoriais com conhecimentos da Física mais avançada. Essa relação, ou melhor interação, somente ocorrerá se os estudantes tiverem conhecimentos sobre números complexos na estrutura cognitiva, caso contrário, dificilmente ocorrerá o processo de interação entre conhecimentos prévios (conhecimentos matemáticos) e novos (conhecimentos de Física). A falta de conhecimentos prévios dificulta a compreensão e a aprendizagem dos conceitos relacionados aos circuitos elétricos. Nesse cenário, o estudante terá dificuldade para compreender os conceitos ou os processos de resolução e, possivelmente, memorizará algoritmos para resolver casos específicos envolvendo os números complexos.

No contexto apresentado em PE5, os estudantes possuem algum conhecimento dos números complexos, porém PE1 e PE2 mostram que os estudantes ou acadêmicos não têm esse conhecimento, dificultando a aprendizagem de circuitos elétricos em corrente alternada. Assim, é imprescindível o professor abordar esse conteúdo, relacionando a representação algébrica com a geométrica para permitir a construção de significados, conforme é exposto na PE3, PE4 PE6 e PE7, e somente os autores da PE6 introduziram os números complexos para estudantes ou acadêmicos que nunca o haviam estudado.

Além disso, observa-se que nas pesquisas predominam ou a problemática de números complexos ou de circuitos elétricos em corrente alternada. Nenhuma das pesquisas descreve o ensino de números complexos para, posterior ou concomitantemente, abordar a análise de circuitos elétricos em corrente alternada, ressaltando a importância dos dois contextos de aprendizagem: o matemático e o físico.

Em relação aos recursos digitais para auxiliar nos processos de ensino e de aprendizagem, as pesquisas que integraram esse mapeamento foram aplicadas a estudantes do Ensino Médio ou do Ensino Médio Técnico. Entre os recursos encontrados destacam-se os objetos de aprendizagem, disponíveis na web, denominados Números Complexos e Números Complexos: 
interação e aprendizagem, respectivas das pesquisas PE4, PE9 e P10. Esses OA contemplam aspectos algébricos e geométricos de números complexos, buscando envolver os estudantes e promover aprendizagens. Apesar disso, observam-se limitações nos OA, em que o Números Complexos: interação e aprendizagem apresentam um texto introdutório relacionando os conhecimentos de números complexos com os circuitos elétricos. Além disso, os OA não foram submetidos a nenhum instrumento para avaliar a sua potencialidade como um recurso digital para qualificar os processos de ensino e de aprendizagem.

Portanto, ao analisar as produções desses periódicos, verificou-se a existência de poucas pesquisas que abordam sobre o ensino e a aprendizagem de números para acadêmicos de Engenharia Elétrica, conforme se pretendeu averiguar e conhecer. Corroborando com a falta de pesquisas dessa temática, Puhl e Müller (2017) apresentam resultados similares, por meio de um mapeamento realizado em eventos nas áreas de ensino de Matemática, ensino de Engenharia e da Informática na Educação.

Diante disso, há a necessidade de investigar essa temática e propor uma alternativa pedagógica para auxiliar os professores e acadêmicos, pois este é um problema presente em outras instituições de Ensino Superior (GERMANO, 2016; JESUS; TESTANI, 2016; AGRICCO JUNIOR, 2017; LIMA, 2017; LINHARES, 2017; PORTOLAN, 2017). Sendo assim, pensa-se em planejar a construção de um OA, disponível virtualmente e de acesso gratuito, que propicie o ensino de números complexos associado à análise de circuitos elétricos em corrente alternada. Esse OA tem como principal público estudantes de nível Médio Técnico, acadêmicos e professores de Engenharia Elétrica. Desse modo, demais professores poderão apresentar seus resultados e seus feedbacks, o que servirá para qualificar continuamente o OA.

Este mapeamento propicia, então, uma melhor compreensão do problema que está sendo investigado e motiva para a continuidade dos estudos, principalmente, porque não se encontrou um recurso disponível virtualmente que propicie a construção de conhecimentos sobre números complexos e circuitos elétricos em corrente alternada. 


\section{Referências}

ABEGG, I.; RAMOS, D. B. Investigação de ferramentas e métodos de ensino de circuitos de corrente alternada para curso introdutório de eletrotécnica. Revista Dynamis, Blumenau/SC, v. 19, n. 1, 2013.

AGRICCO JUNIOR, R. C. Números complexos e grandezas elétricas: análise de livros didáticos apoiada na teoria dos registros de representações semióticas. 2017. Dissertação (Mestrado) — Universidade Anhanguera de São Paulo, São Paulo, 2017.

ALVES, F. R. V. Transição complexa do cálculo - TCC: engenharia didática para as noções de sequências, séries e série de potências. Educação Matemática em Revista, Canoas/RS, v. 1, n. 17, 2016.

ALVES-MAZZOTTI, A. J.; GEWANDSZNAJDER, F. O método nas ciências naturais e sociais: pesquisa quantitativa e qualitativa. 2. ed. São Paulo: Pioneira Thomson Learning, 1999.

BATISTA, S. C. F. SOFTMAT: um repositório de softwares para matemática do Ensino Médio - um instrumento em prol de posturas mais conscientes na seleção de softwares educacionais. 2004. Dissertação (Mestrado) — Universidade Estadual do Norte Fluminense, Campos dos Goytacazes, 2004.

BATISTA, S. C. F. et al. Investigando em C: uma unidade de aprendizagem online para estudo de números complexos. RENOTE - Revista Novas Tecnologias na Educação, Porto Alegre/RS, v. 7, n. 1, 2009.

BIEMBEnGUT, M. S. Mapeamento na Pesquisa Educacional. Rio de Janeiro: Ciência Moderna, 2008.

BITENCOURT, A. L.; VARGAS, P. R.; FELICETTI, V. L. Una propuesta pedagógica: utilizando el software geogebra en la rotación de vectores complejos. Amazônia - Revista de Educação em Ciências e Matemáticas, Belém/PA, v. 11, n. 21, 2014.

BRASIL, Ministério da Educação. Pacto Nacional pelo Fortalecimento do Ensino Médio. Brasília: MEC/SEB, 2013.

BRASIL, Ministério da Educação. Programa Ensino Médio Inovador: documento orientador. Brasília: MEC/SEB, 2014.

BRASIL, Ministério da Educação. Base Nacional Comum Curricular: Educar é a base. Brasília: MEC, 2018.

COELHO, M. C. B. Números Complexos e suas aplicações geométricas no ensino superior. 2013. Dissertação (Mestrado Profissional) - Centro Federal de Educação Tecnológica Celso Suckow da Fonseca, Rio de Janeiro, 2013.

COSTA, J. C. Números Complexos: uma abordagem com ênfase em aplicações na matemática e em outras áreas. 2016. Dissertação (Mestrado Profissional) - Universidade Federal do Maranhão, São Luís, 2016.

CRESWELL, J. W. Projeto de Pesquisa: Métodos qualitativo, quantitativo e misto. 3. ed. Porto Alegre: Artmed, 2010. 
ELI, J. Números complexos e suas aplicações: uma proposta de ensino contextualizado com abordagem histórica. 2014. Dissertação (Mestrado) - Universidade Regional de Blumenau, Blumenau, 2014.

GERMANO, J. G. C. Uma proposta de abordagem dos Números Complexos com o uso do Geogebra. 2016. Dissertação (Mestrado Profissional) - Universidade Federal do Ceará, Fortaleza, 2016.

GIL, A. C. Como elaborar projetos de pesquisa. 6. ed. São Paulo: Atlas, 2017.

JESUS, M. A. S.; TESTANI, G. B. As atitudes em relação à matemática e o desempenho em cálculo diferencial e integral na variável complexa. In: ENCONTRO NACIONAL DE EDUCAÇÃO MATEMÁTICA, 12., 2016. Anais [... ]: São Paulo/SP, 2016.

LIMA, H. M. Aplicação de números complexos em circuitos elétricos. 2017. Dissertação (Mestrado Profissional) — Universidade Federal do ABC, Santo André, 2017.

LINHARES, M. F. Análise dos Ambientes Virtuais de Aprendizagem (AVA's) AulaNet, Moodle e TelEduc e implementação do ambiente Moodle na Universidade Santa Úrsula. 2017. Dissertação (Mestrado) — Universidade Santa Úrsula, Rio de Janeiro, 2017.

LIRA, E. S. Uma aplicação dos números complexos no Ensino Médio Técnico da Educação Profissional. 2014. Dissertação (Mestrado) — Universidade Federal do Piauí, Teresinha, 2014.

MELLO, S. Q.; SANTOS, R. P. O ensino de matemática e a educação profissional: a aplicabilidade dos números complexos na análise de circuitos elétricos. Acta Scientiae, Canoas, v. 7, n. 2, 2005.

MONZON, L. W.; GRAVINA, M. A. Uma introdução às funções de variável complexa no ensino médio: uma possibilidade através do uso de animações interativas. Bolema, Rio Claro/SP, v. 27, n. $46,2013$.

OLIVEIRA, W. G. A. Estudo e Aplicações dos Números Complexos: O uso dos Números Complexos na Análise de Circuitos Elétricos. 2018. Dissertação (Mestrado) - Pontifícia Universidade Católica do Rio de Janeiro, Rio de Janeiro, 2018.

PARDO T.; GÓMEZ, B. La enseñanza y el aprendizaje de los números complejos: un estudio en el nivel universitário. PNA - Revista de Investigación en Didáctica de la Matemática, Granada - Espanha, v. 2, n. 1, 2007.

PEREIRA, F. O. Números Complexos na Educação Básica. 2016. Dissertação (Mestrado) Universidade Federal do Estado do Rio de Janeiro, Rio de Janeiro, 2016.

PINTO, J. E.; LAUDARES, J. B. Objeto de aprendizagem de números complexos com aplicações na área técnica em eletroeletrônica. Revista Brasileira de Ensino de Ciência e Tecnologia, Ponta Grossa/PR, v. 9, n. 3, 2016.

PORTOLAN, J. A importância do ensino de números complexos no Ensino Médio, na visão dos professores de matemática, em alguns municípios da região oeste do Paraná. 2017. Dissertação (Mestrado) — Universidade Tecnológica Federal do Paraná, Pato Branco, 2017.

PUHL, C. S. Números complexos: interação e aprendizagem. 2016. Dissertação (Mestrado Profissional) - Universidade de Caxias do Sul, Caxias do Sul, 2016. 
PUHL, C. S.; LIMA, I. G. de. Interagindo com os números complexos: revivendo o problema das raízes sofistas. Perspectivas da Educação Matemática, Campo Grande/MS, v. 10, n. 24, 2017.

PUHL, C. S.; LIMA, I. G. de. Números complexos: interação e aprendizagem. Educação Matemática em Revista, Brasília/DF, v. 22, n. 55, 2017.

PUHL, C. S.; MÜLLER, T. J. Mapeamento em anais de eventos: a busca por objetos de aprendizagem para o ensino de números complexos na engenharia elétrica. Revista Eletrônica de Matemática (REMAT), Bento Gonçalves, v. 3, n. 2, 2017.

REIS, A. C. M. A Aplicação dos Números Complexos aos Circuitos de Corrente Alternada no Ensino Técnico: Uma análise no Instituto Federal de Educação, Ciência e Tecnologia do Piauí (IFPI). 2009. Dissertação (Mestrado) - Universidade Luterana do Brasil, Canoas, 2009.

STUMP, S. M.; ABAR, C. A. A. P. Objetos de aprendizagem para ensino de circuitos elétricos em regime estacionário com o uso de números complexos em um curso de engenharia elétrica. In: CONGRESSO INTERNACIONAL EM EDUCAÇÃO EM ENGENHARIA E COMPUTAÇÃO, 8., 2013, Luanda - Angola. Anais [... ]: Luanda - Angola, 2013. 